\title{
Cahiers balkaniques
}

Balkaniques

46 | 2020

Politique et sociétés à Chypre aujourd'hui

Odette VARON-VASSARD, 2019, Des Sépharades aux juifs grecs. Histoire, mémoire et identité, Le Manuscrit, Paris, 226 p., ISBN : 9782304047523

Hervé Georgelin

\section{(2) OpenEdition}

Édition électronique

URL : https://journals.openedition.org/ceb/15333

DOI : $10.4000 /$ ceb.15333

ISSN : 2261-4184

Éditeur

INALCO

Édition imprimée

ISBN : 9782858313341

ISSN : 0290-7402

Référence électronique

Hervé Georgelin, «Odette varon-vassard, 2019, Des Sépharades aux juifs grecs. Histoire, mémoire et identité, Le Manuscrit, Paris, 226 p., ISBN : 9782304047523 », Cahiers balkaniques [En ligne], 46 | 2020, mis en ligne le 08 janvier 2020, consulté le 06 juillet 2021. URL : http://journals.openedition.org/ceb/ 15333 ; DOI : https://doi.org/10.4000/ceb.15333

\section{(c) (1) \&}

Cahiers balkaniques est mis à disposition selon les termes de la Licence Creative Commons Attribution - Pas d'Utilisation Commerciale 4.0 International. 


\title{
Odette Varon-Vassard, 2019, Des Sépharades aux juifs grecs. Histoire, mémoire et identité, Le Manuscrit, Paris, 226 p., ISBN : 9782304047523
}

\author{
Hervé Georgelin \\ Université d'Athènes, Département d'Études turques
}

À partir de textes rédigés entre 2003 et 2018, l'auteure nous présente son vade-mecum pour appréhender l'histoire des Juifs de Grèce. Qu'est-ce donc que le point de vue historien adopté par Varon-Vassard? Il s'agit d'un regard qui est sensible aux transformations à travers le temps, mais aussi aux nuances d'un phénomène dans un espace donné. Si ce regard est sensible aux transformations, il doit également saisir les occurrences de permanence, puisqu'il s'agit des deux rapports possibles au temps des choses humaines. Il n'y a généralement jamais de complète continuité en histoire. Il n'y a jamais non plus de tabula rasa absolue, même si certains acteurs historiques peuvent prétendre ou viser le contraire.

Les juifs de Grèce aujourd'hui, discrets, peu nombreux, hellénophones, imperceptibles dans le tissu urbain d'Athènes ou de Thessalonique désormais, pour prendre les communautés les plus importantes, sont en fait les héritiers d'une continuité séculaire de présence juive à l'extrémité méridionale de la péninsule balkanique, mais de ruptures majeures et notamment de celle qui a bien failli leur coûter leur complète existence collective, soit l'occupation nazie et le plan d'extermination des juifs d'Europe qui caractérise le Troisième Reich, partout où il a gouverné ou occupé.

Un regard d'historien, c'est aussi le souci de l'exactitude des notes de bas de page, c'est-à-dire de l'usage de références, car, à la différence de l'écriture littéraire fictionnelle, l'écriture en sciences sociales se doit de répondre d'elle-même. Elle ne se déploie pas dans le silence de la profession, rares sont les sujets pour lesquels l'historien n'a ni prédécesseur ni collègue contemporain, éventuellement concurrent. Il y a donc des références qu'un historien lisant un livre d'histoire attend, à un tournant de page, à un moment ou un autre pour s'assurer que le 
nouveau texte s'insère dans une discussion déjà entamée, au moins en partie. Les références sont là, elles défilent et contentent le regard en éveil du lecteur informé: Aron Rodrigue, Mina Rozen, Haïm Vidal Séphiha, pour n’en citer que quelques-uns.

L'écriture historienne de Varon-Vassard ne se déploie pas non plus dans le silence des gens étudiés, car ceux-ci ont généralement laissé des traces qui deviennent les sources primaires de l'écrivant historien. Le livre de Varon-Vassard n'est pas non plus étranger aux écritures littéraires même fictionnelles des époques qu'elle prend pour sujet. Il y a parfois dans des récits de fiction plus de vérité que dans la presse, par exemple. À propos de littérature, Odette Varon a au moins une seconde corde à son arc. Parallèlement à ses activités d'historienne inaugurée par un doctorat en histoire à l'université d'Athènes, soutenu en 1995, portant sur les organisations de jeunesse de la résistance grecque, Odette Varon-Vassard a

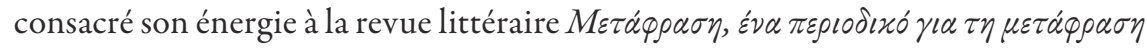
de 1995 à 2007. Elle a aussi pratiqué elle-même la traduction littéraire, du français vers le grec moderne. On retrouvera des thématiques qui ont à voir, directement ou par analogie, avec le sujet du présent livre, comme, par exemple: en 2018, de David Foenkinos, Charlotte, publiée chez Hestia, ou d'Albert Cohen, Solal, publiée en 1992 (une édition révisée paraîtra fin 2019 aux éditions Exandas) chez Chatzinikoli et Mangeclous, publié chez Iridanos en 1994.

Des Sépharades aux juifs grecs. Histoire, mémoire et identité fait le point de façon synthétique sur son propre parcours d'historienne des juifs de l'aire qui actuellement constitue le territoire de la Grèce moderne, mais aussi des apports de ses collègues. Odette Varon donne aussi la parole aux voix des écrivains qui sont, soit issus de la population juive de Grèce comme Albert Cohen, juif corfiote et écrivain francophone; soit ceux qui se trouvaient être leurs voisins, comme Yorgos Ioannou dont l'attachement à certains de ses voisins à Thessalonique était profond, lié à sa découverte des dimensions érotiques de l'existence.

L'auteur n'a jamais refusé la Grèce moderne, dans laquelle elle s'est retrouvée à sa naissance, elle aussi juive de Grèce, dans un contexte marqué par la disparition, l'euphémisme perécien est désormais connu, de la majeure partie de ses coreligionnaires potentiels. Il peut être plus facile de comprendre ceux des survivants en 1945 qui prirent la direction de l'exil radical vers l'Israël en devenir ou l'Occident, plutôt que de rentrer dans des lieux où on ne pouvait pas réellement revenir, comme la Thessalonique, désormais hellénisée, semblable à un désert, un Sahara humain pour les quelques milliers qui y passèrent, soit après avoir été cachés, soit après avoir survécu à l'enfer des camps. Mais à l'inverse, comment comprendre que l'exil radical n'apporte pas forcément non plus la réparation? À cet égard, l'ouvrage d'Aharon Appelfeld (né aux confins de la Roumanie et de 
l'Ukraine, à Tschernowitz, qui aurait dû devenir un intellectuel germanophone à l'instar de Paul Celan), Le Garçon qui voulait dormir, est édifiant quant à la difficulté d'adaptation d'un jeune rescapé sur les rives de la Méditerranée orientale. D’une façon générale, toute l'œuvre de l'écrivain israélien, bien qu'écrite en hébreu, ne parle presque que de l'Europe d'avant-guerre ou du conflit, comme si l'œuvre littéraire n'était qu'un travail de deuil infini. Les dilemmes ashkénazes ne sont pas bien éloignés de ceux que juifs sépharades ou romaniotes de Grèce ont dû affronter en 1945 et dans les années qui suivirent. Où se sentir chez soi sinon dans les lieux, dans les langues qui nous ont portés depuis toujours, malgré la trahison de certains, de beaucoup, de bien trop des non-juifs?

La prédilection pour le français qu'Odette Varon-Vassard illustre par la publication de ce livre dans cette langue est aussi un signe d'affiliation au passé sépharade de Salonique, où les écoles de l'Alliance israélite universelle préparaient ses élèves à entrer dans la vie économique de la cité, en réseau avec les autres centres économiques de la Méditerranée occidentale qui avaient également le français comme langue-outil de communication et comme critère de distinction culturelle et sociale. Ce livre en français nous rappelle ainsi que cette langue n'a pas pour essence d'être un détail ethnologique d'un monde globalisé qui la marginalise, mais qu'elle est, en puissance, tout à fait capable elle aussi d'incarner la contemporanéité. Les textes des historiens eux-mêmes deviennent des traces, des témoignages de leur propre temps. Il y aura peu d'historiens grecs qui écriront en français à l'avenir.

Le livre d'O. Varon-Vassard fait également le point, en français, sur la bibliographie actuelle du champ d'études, c'est-à-dire sur l'état de la recherche sur les juifs de Grèce, tout en accordant une place de choix à la rupture principale de cette histoire, l'extermination des juifs par l'Allemagne nazie, secondée dans son œuvre par l'occupant bulgare et les collaborateurs grecs. Cet ouvrage est à ce titre un important outil pour tous les étudiants dominant le français qui s'intéressent à la Grèce contemporaine. À cet égard, signalons le fait que les étudiants grecs actuels d'une vingtaine d'années ignorent souvent la diversité et l'ancienneté de la présence juive sur le territoire de la Grèce contemporaine. Ils n'ont généralement qu'une très vague idée des effectifs de la communauté juive actuelle ou de la relativité de la catégorie « juif ». (Qui l'est? Qui ne l'est pas? L'est-on un peu? Est-ce une propriété scalaire ?) Il y a beaucoup à faire pour préciser et affiner la perception de leur propre espace. Un ouvrage comme celui d' Odette Varon-Vassard, s'il leur était accessible, pourrait y contribuer.

Revenons sur le titre qui met en exergue un phénomène historique important pour la population juive en Grèce comme ailleurs en Europe, bien que selon des chronologies différentes. Il s'agit de la transformation d'une communauté 
religieuse, perçue par la population tutélaire, mais aussi très souvent revendiquée par le groupe concerné lui-même comme fondamentalement autre et relevant d'un ailleurs: la Palestine biblique ou l'Espagne médiévale, comme c'était le cas des Sépharades de Grèce. L'effacement du régime d'altérité radicale, juridique, mais aussi peut-être anthropologique était en Europe occidentale en marche depuis l'époque des Lumières, avec en France une accélération lors de la Révolution et l'Empire. Les juifs français ou prussiens, par exemple, accédèrent à la citoyenneté, en même temps qu'elle se généralisait pour les autres également. Force est de constater que le processus, en particulier à Thessalonique, n'était pas achevé lors de l'occupation de la Grèce par le III $^{\mathrm{e}}$ Reich et ses alliés. Les juifs de Salonique et de Thrace ou de Macédoine, venus à la fin du Xv siècle de l'Espagne reconquise par le catholicisme, avait trouvé refuge dans l'Empire ottoman qui certes les accepta comme tels, mais les soumit à la règle de la Zimmet (en $\operatorname{turc}^{1}$ ) (en arabe ${ }^{2}:$ o c'est-à-dire à un régime d'infériorité légale, morale, juridique et politique qui leur laissait la vie sauve.

La conquête de la Salonique cosmopolite ou de la Selanik ottomane par la Grèce contemporaine en 1912 devait ouvrir la voie à la transformation radicale de la cité en Thessalonique néogrecque. Si les juifs ottomans ou étrangers qui résidaient sur place désiraient y demeurer, ils devaient s'accommoder du processus qui, du moins formellement, leur accordait pleine égalité de droits avec le reste des citoyens grecs. Ces intentions n'ont pas été réalisées du jour au lendemain. Sous la rhétorique intégrationniste, des visées tout autres étaient dissimulées, comme le prouve le réagencement de l'espace urbain après l'incendie accidentel de 1917 qui permit la marginalisation spatiale de la communauté juive. A contrario, il était difficile pour certains juifs saloniciens de renoncer à une éducation francophone, c'est-à-dire d'entretenir un horizon mental, culturel et économique qui ne coïncidait pas avec celui que l'État-nation hellénique offrait, forcément plus étroit. Ce qui vaut pour le français, lingua franca prestigieuse de la Méditerranée orientale jusqu'à la fin de la Seconde Guerre mondiale, vaut a fortiori pour le judéo-espagnol, langue de l'intimité mais dépourvue de prestige et de valeur d'échange hors de la communauté sépharade, qui devenait stigmate après l'arrivée des Grecs-orthodoxes d'Asie Mineure, eux-mêmes hellénophones ou turcophones, et le départ des musulmans saloniciens échangés. De la cité ottomane cosmopolite, il ne restait plus grand-chose et ce passé était honni par ses nouveaux maîtres.

1. https://www.nisanyansozluk.com/?k=zimmet

2. https://www.lescahiersdelislam.fr/glossary/Dhimma-\%D8\%B0\%D9\%85\%D8\%A9_ gw137.html 
L'histoire de Salonique est évoquée avec force détails par Varon-Vassard. L'auteure est sans doute, de son propre aveu, moins diserte à propos des communautés romaniotes, en particulier celle de Ioannina, mais également d'Arta et Patras. Néanmoins, chaque lieu, de Komotiní à Katerini, de Corfou à Rhodes est évoqué dans le livre. La Canée est même en couverture du livre. La prépondérance de Salonique, Madre De Israel, aux 90000 juifs à la fin de l'Empire ottoman justifie la place qu'elle prend dans le panorama offert par Odette Varon-Vassard.

Concluons en disant que ce livre est fort riche et qu'il fait le point sur la bibliographie actuelle la plus récente. Elle intègre par exemple Luna de Rika Benveniste, paru en 2018. Le livre conjugue les faits et les représentations, en intégrant la littérature à son propos. Il remet, en perspective chiffrée, l'importance des communautés partout en Grèce, avant l'occupation nazie et leur effectif actuel quand elles ont pu se reconstituer. À tout point de vue, cet ouvrage replace les juifs sur la carte de la Grèce contemporaine et sur celle du judaïsme européen qu'on assimile souvent exclusivement au monde ashkénaze. C'est un livre précieux qui souligne aussi les efforts faits récemment par certaines instances grecques modernes pour intégrer ce passé et ce ténu présent à la perception générale de l'histoire du pays, pensons au projet du maire de Thessalonique (2010-2019), Yannis Boutaris, qui a lancé le projet d'un musée de la Shoah dans sa ville. Il n'est que peu d'activité historienne dénuée de dimension politique. 